\title{
Analytic analysis of LTE/LTE-Advanced power saving and delay with bursty traffic
}

\author{
R. S. Bhamber, Scott Fowler, C. Braimiotis and A. Mellouk
}

\section{Linköping University Post Print}

\section{Tweet}

N.B.: When citing this work, cite the original article.

(C2013 IEEE. Personal use of this material is permitted. However, permission to reprint/republish this material for advertising or promotional purposes or for creating new collective works for resale or redistribution to servers or lists, or to reuse any copyrighted component of this work in other works must be obtained from the IEEE.

R. S. Bhamber, Scott Fowler, C. Braimiotis and A. Mellouk, Analytic analysis of LTE/LTEAdvanced power saving and delay with bursty traffic, 2013, IEEE International Conference on Communications (ICC'13), (), , 2964-2968.

http://dx.doi.org/10.1109/ICC.2013.6654993

Postprint available at: Linköping University Electronic Press http://urn.kb.se/resolve?urn=urn:nbn:se:liu:diva-107929 


\title{
Analytic Analysis of LTE/LTE-Advanced Power Saving and Delay with Bursty Traffic
}

\author{
Ranjeet S. Bhamber*, Scott Fowler ${ }^{\dagger}$, Christos Braimiotis and Abdelhamid Mellouk ${ }^{\S}$ \\ *Instituto de Óptica "Daza de Valds", C.S.I.C. 121, Serrano, 28006 Madrid, Spain \\ ${ }^{\dagger}$ Mobile Telecommunications, Department of Science and Technology Linköping University, Norrköping, Sweden \\ ${ }^{\S}$ LiSSi laboratory, Department of Networks and Telecommunication, IUT C/V University of Paris-Est Creteil (UPEC), France
}

\begin{abstract}
The 4G standard Long Term Evolution (LTE) has been developed for high-bandwidth mobile access for today's data-heavy applications. However, these data-heavy applications require lots of battery power on the user equipment. To extend the user equipment battery lifetime, plus further support various services and large amount of data transmissions, the 3GPP standards for LTE/LTE-Advanced has adopted discontinuous reception (DRX). In this paper, we take an overview of various static/fixed DRX cycles of the LTE/LTE-Advanced power saving mechanisms, by modelling the system with bursty packet data traffic using a semi-Markov process. Based on the analytical model, we will show the trade-off relationship between the power saving and wake-up delay performance. This work will help to select the best parameters when LTE/LTE-Advanced DRX is implemented depending on the protocols and desired outcome of the traffic.
\end{abstract}

\section{INTRODUCTION}

The advancement of mobile technologies has profoundly affected our lives. It is a rapidly growing trend that more users are becoming dependent on the mobile tools as their primary computing devices and replacing the traditional stationary hardware. We see a variety of powerful smart mobile devises (e.g. iPhone, iPad, Tablets) handling a wide range of traffic including multimedia. Thus, a 4G (fourth generation) standard, LTE/LTE-Advanced (henceforth referred to as LTE) has been developed that is intended for larger capacity and higher speed of mobile networks. Even though mobile hardware keeps evolving, they will always be resource-poor relative to stationary hardware. The reason is that, first, battery technologies for mobile devices only allow limited computing power on a portable-lightweight package, and second, the processing power and the memory of mobile hardware are much smaller than those of traditional desktops and laptops. This presents a challenge for a mobile device to execute resource-hungry user applications.

To extend the user equipment battery lifetime, plus further support various services and large amount of data transmissions, the 3GPP standards for LTE has adopted DRX and Discontinuous Transmission (DTX) power-saving mechanisms protocols, thereby providing energy-efficient-Green Network. The theoretical basis of traditional scheduling mechanisms becomes invalid when DRX is adopted. To address this problem there is a need to optimize the DRX parameters, so as to maximize power saving without incurring network re-entry and packet delays. In particular, care should be exercised for real-time services.

In this paper, we take an overview of the fixed/static DRX cycles with a semi-Markov process in order to evaluate the power saving and wake-up delay performance of LTE DRX mechanisms. The results show that there is a trade-off relationship between the power saving and wake-up delay performance for various fixed/static DRX parameters. This work will help to select the best parameters when LTE DRX is implemented.

\section{LTE AND THE DRX CONCEPT}

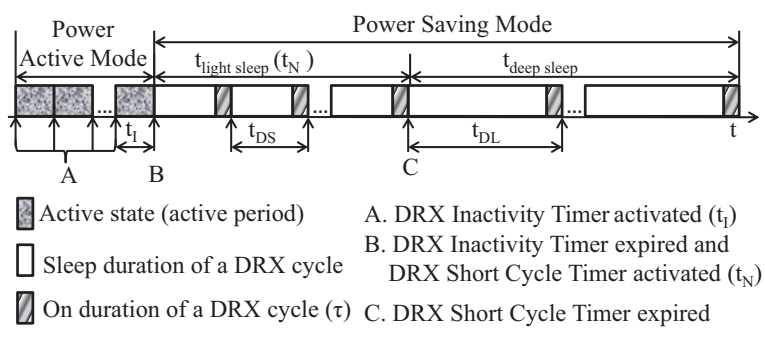

Fig. 1: LTE DRX timing for UE receiver operations.

LTE's energy efficient strategy exploits the concepts of DRX and DTX [2], [4]. In the LTE DRX mechanism, the sleep/wake scheduling of each User Equipment (UE) is determined by the following four parameters [10] ${ }^{1}$ DRX Short Cycle $\left(t_{D S}\right)$, DRX

${ }^{1}$ previous work focused on a 3-state adjustable cycles, while this work focues on 4-state fixed cycles 
Long Cycle $\left(t_{D L}\right)$, DRX Inactivity Timer $\left(t_{I}\right)$ and DRX Short Cycle Timer $\left(t_{N}\right)$ as shown in Figure 1 . The $t_{D S}$ and $t_{D L}$ define duration of OFF and ON period, which is a fixed/static value applied to both long and short cycles. UE monitors the physical downlink control channel $(\mathrm{PDCCH})$ to determine if there is any transmission over the shared data channel allocated to the UE during ON duration. The $t_{I}$ specify the period where UE should stay awake and monitor PDCCH after the last successful decoding of PDCCH. The $t_{N}$ specifies the period where UE should follow $t_{D S}$ after the $t_{I}$ has expired.

In LTE DRX, the sleep/wake-up mode consists of the three different states, namely, Inactivity period, Light Sleep period, and Deep Sleep period. The Inactivity period is the power active mode, whereas the Light Sleep period and the Deep Sleep period are the power saving mode. The transition from the Inactivity period to the Light Sleep period is controlled by $t_{I}$, while the transition from the Light Sleep period to the Deep Sleep period within the power saving mode is controlled by $t_{N}$.

The following describes how the UE works during the Inactivity, Light Sleep, and Deep Sleep periods [1].

DRX Inactivity period: Is when the DRX Inactivity Timer $^{2}$ is $\mathrm{ON}$, and the UE receiver is monitoring the PDCCH, while being ready to receive packets through the evolved node-B (eNB) from Evolved Packet Core (EPC). Should the DRX Inactivity Timer expire, then the DRX Short Cycle Timer is activated and the Light Sleep period begins.

DRX Light Sleep period: Consists of the DRX Short Cycles $\left(t_{D S}\right)$. During each of the DRX Short Cycle the UE wakes up to monitor the PDCCH (also know as Listen Interval). If the PDCCH indicates a downlink transmission, the UE changes to an activity period and starts the $t_{I}$. Otherwise the UE will return to Light Sleep period. The UE will keep entering Light Sleep period until the DRX Short Cycle Timer $^{3}$ expires.

DRX Deep Sleep period: During each of the DRX Deep Long Cycle the UE wakes up to monitor the PDCCH. If the PDCCH indicates a downlink transmission, the UE changes from Deep Sleep period to activity period and starts the DRX Inactivity Timer. Otherwise, the UE will return to Deep Sleep.

\section{An Analytical Model For LTE Power SAVing}

\section{A. Bursty Packet Traffic Model}

Studies have shown that for some environments, the traffic data are self-similar [12] rather than the traditional queuing that is contingent on the data traffic to be Poisson as mentioned in [10]. In the traditional Poisson Traffic model, it usually has a very limited range of time scales and making it short range dependent. Self-similar traffic displays burstiness and interacts over an immensely wide range of time scales and making it long range dependent. In addition, it has been shown that heavy tailed such as Pareto and Weibull distributions are more applicable when modelling data network traffic [7]. For this paper, we used the European Telecommunication Standards Institute (ETSI) traffic model [3], where the packets size and the packet transmission timer are assumed to follow the truncated Pareto distribution. The ETSI model is a widely used in various analytical and simulation studies of 3GPP networks, such as [5], [8], [11], [15], [16]. [7] shows that the $M / G / \infty$ model with infinite-variance Pareto distributions can be used to generate self-similar traffic.

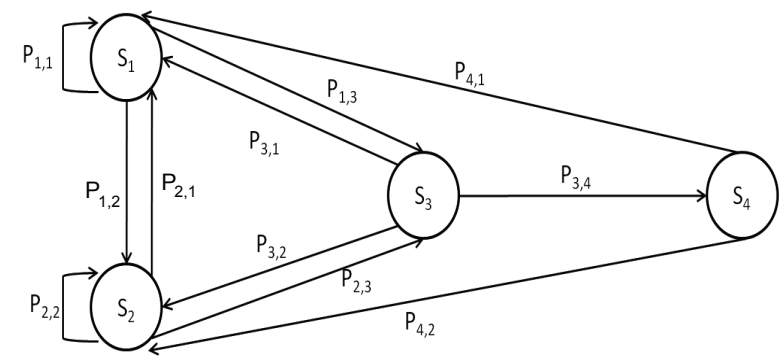

Fig. 2: 4-State semi-Markov process for LTE DRX.

The LTE DRX mechanism is a semi-Markov process [9] and is illustrated in Figure 2. The state transition diagram consists of four states ${ }^{4}$, which are relevant to the three periods shown in Figure 1.

- State $S_{1}$ comprise a busy/active period $t_{B}^{*}$ (Power Active Mode) and inter-packet call inactivity period $t_{I 1}^{*}$.

- State $S_{2}$ comprise a busy/active period $t_{B}^{*}$ (Power Active Mode) and inter-session inactivity period $t_{I 2}^{*}$.

- State $S_{3}$ comprises a Light Sleep period ( $t_{\text {light sleep }}$ ) which is entered from $S_{1}$ or $S_{2}$.

- State $S_{4}$ comprises a Deep Sleep period $\left(t_{\text {deep sleep }}\right)$ which is entered from $S_{3}$.

${ }^{2}$ Inactivity Timer: Specifies the number of consecutive TTIs during which UE shall monitor PDCCH after successfully decoding a PDCCH indicating a UL or DL data transfer for this UE.

${ }^{3}$ DRX Short Cycle Timer $\left(t_{N}\right)$ : Indicates the number of initial DRX cycles to follow the short DRX cycle before transitioning to the long DRX cycle.

${ }^{4}$ Even combining $S_{1}$ and $S_{2}$, we had the same results. However, by separating the "Powering Active Mode" ( $S_{1}$ and $\left.S_{2}\right)$, it will provide future research on the behaviour of energy saving when $t_{I}$ is small. 
A new packet call can be viewed as continuation of the current session or as the onset of a new session depending on the time interval-arrive between two consecutive packet calls. The packet calls may be the inter-packet call idle time $\left(t_{i p c}\right)$ with probability $1-1 / \mu_{p c}$ or the inter-session idle time $\left(t_{i s}\right)$ with probability $1 / \mu_{p c}$. The probabilities take into account the memoryless property of a geometric distributions. If we view this semi-Markov process only at the times of state transitions, we obtain an embedded Markov chain with state transition probabilities $P_{i, j}$, where $i, j \in\{1,2,3,4\}$.

\section{B. State 1 to State 1 , State 1 to State 2 and State 1 to State 3}

In state $S_{1}$, the RNC inactivity timer is activated at the end of the busy period $t_{B}^{*}$, and then the UE enters the DRX Inactivity period $t_{I 1}$. When the first packet of the next call arrives at the RNC before the DRX Inactivity timer expires, with a probability of $q_{1}=\operatorname{Pr}\left[t_{i p c}<t_{I}\right]=1-\mathrm{e}^{-\lambda_{i p c} t_{I}}$, the timer is stopped, and another busy period begins. In this case, if the new arriving packet call is the last one of the ongoing session (with probability $1 / \mu_{p c}$ ), then the UE enters state $S_{2}$, otherwise with the probability $1-1 / \mu_{p c}$ the ongoing session continues, and the UE enters state $S_{1}$ again. This gives us:

$$
\begin{gathered}
p_{1,1}=\left(1-e^{-\lambda_{i_{p c} t_{I}}}\right)\left(1-\frac{1}{\mu_{p c}}\right)=q_{1}\left(1-q_{2}\right) \\
p_{1,2}=\left(1-e^{-\lambda_{i p c} t_{I}}\right)\left(\frac{1}{\mu_{p c}}\right)=q_{1} q_{2}
\end{gathered}
$$

If no packets arrives before the inactivity timer expires, then the UE enter into light sleep:

$$
p_{1,3}=\left(e^{-\lambda_{i p c} t_{I}}\right)=1-q_{1}
$$

\section{State 2 to State 1, State 2 to State 2 and State 2 to State 3}

The derivations of $p_{2,1}$ and $p_{2,2}$ are exactly the same as that of $p_{1,1}$ and $p_{1,2}$ except that the inter-packet call idle period $t_{i p c}$ is replaced by the inter-session idle period $t_{i s}$ and $q_{1}$ is replaced by $q_{3}=\operatorname{Pr}\left[t_{i s}<t_{I}\right]=1-\mathrm{e}^{-\lambda_{i s} t_{I}}$. Therefore, we have:

$$
\begin{aligned}
& p_{2,1}=\left(1-e^{-\lambda_{i s} t_{I}}\right)\left(1-\frac{1}{\mu_{p c}}\right)=q_{3}\left(1-q_{2}\right) \\
& p_{2,2}=\left(1-e^{-\lambda_{i s} t_{I}}\right)\left(\frac{1}{\mu_{p c}}\right)=q_{2} q_{3}
\end{aligned}
$$

Similarly, $p_{2,3}$ can be derived by substituting $q_{3}$ for $q_{1}$ in Equation (3), we have:

$$
p_{2,3}=\left(e^{-\lambda_{i s} t_{I}}\right)=1-q_{3}
$$

\section{State 3 to State 1, State 3 to State 2 and State 3 to State 4}

In state $S_{3}$, the UE follows DRX Short Cycles with the probability that there is at least one initiation of awakening during Inter-packet call is $1-e^{-\lambda_{i p c} t_{N}}$. If the PDCCH indicates that a new packet call starts before the DRX Short Cycle Timer expires (means new packet call occurs before $t_{N}$ has expired), the timer is cancelled. If the next packet call terminates the ongoing session (with probability $q_{2}$ ), then the UE will move to $S_{2}$ in the next transition. Otherwise (with probability 1 - $q_{2}$ ), the UE will change to $S_{1}$. Thus

$$
\begin{gathered}
p_{3,1}=\left(1-e^{-\lambda_{i p c} t_{N}}\right)\left(1-\frac{1}{\mu_{p c}}\right)=q_{4}\left(1-q_{2}\right) \\
p_{3,2}=\left(1-e^{-\lambda_{i s} t_{N}}\right)\left(\frac{1}{\mu_{p c}}\right)=q_{2} q_{5}
\end{gathered}
$$

If the PDCCH indicates that there is no packet call delivery happening after the DRX Short Cycle Timer expires (meaning no new packet during the DRX Short Cycle times $t_{N}$ ), then $S_{4}$ is entered:

$$
\begin{aligned}
p_{3,4} & =\left(e^{-\lambda_{i p c} t_{N}}\right)\left(1-\frac{1}{\mu_{p c}}\right)+\left(e^{-\lambda_{i s} t_{N}}\right)\left(\frac{1}{\mu_{p c}}\right) \\
& =\left(1-q_{2}\right)\left(1-q_{4}\right)+q_{2}\left(1-q_{5}\right)
\end{aligned}
$$

\section{E. State 4 to State 1 and State 4 to State 2}

In state $S_{4}$, if the next packet call terminates the ongoing session (with probability $q_{2}$ ), then the UE will move to $S_{2}$ in the next state transition. Otherwise, (with probability $1-q_{2}$ ), the UE will switch to state $S_{1}$. This gives us:

$$
\begin{gathered}
p_{4,1}=\left(1-\frac{1}{\mu_{p c}}\right)=1-q_{2} \\
p_{4,2}=\left(\frac{1}{\mu_{p c}}\right)=q_{2}
\end{gathered}
$$




\section{F. Transition Probability Matrix}

The transition probability matrix $\mathbf{P}=\left(P_{i, j}\right)$ of the embedded Markov chain can, hence, be given as (12):

$$
\mathbf{P}=\left[\begin{array}{cccc}
P_{1,1} & P_{1,2} & P_{1,3} & 0 \\
P_{2,1} & P_{2,2} & P_{2,3} & 0 \\
P_{3,1} & P_{3,2} & 0 & P_{3,4} \\
P_{4,1} & P_{4,2} & 0 & 0
\end{array}\right]
$$

Let $\pi_{i}(i \in\{1,2,3,4\})$ denote the probability that the embedded Markov chain is in state $S_{i}(i \in\{1,2,3,4\})$. By using $\sum_{j=1}^{4} \pi_{i}=1$ and the balance equation $\pi_{i}=\sum_{j=1}^{4} \pi_{j} P_{j, i}$, we can solve the stationary distribution and obtain (13)

$$
\Pi= \begin{cases}\pi_{1}= & \frac{\left(1-q_{2}\right)\left(1+q_{2}\left(1-q_{3}\right)\left(q_{4}-q_{5}\right)\right)}{1+\left(1-q_{2}\right)\left(1-q_{1}\right)\left(2-q_{4}\right)+q_{2}\left(2-q_{5}\right)\left(1-q_{3}\right)} \\ \pi_{2}= & \frac{q_{2}\left(1-\left(1-q_{1}\right)\left(1-q_{2}\right)\left(q_{4}-q_{5}\right)\right)}{1+\left(1-q_{2}\right)\left(1-q_{1}\right)\left(2-q_{4}\right)+q_{2}\left(2-q_{5}\right)\left(1-q_{3}\right)} \\ \pi_{3}= & \frac{\left(1-q_{1}\right)\left(1-q_{2}\right)+q_{2}\left(1-q_{3}\right)}{1+\left(1-q_{2}\right)\left(1-q_{1}\right)\left(2-q_{4}\right)+q_{2}\left(2-q_{5}\right)\left(1-q_{3}\right)} \\ \pi_{4}= & \frac{\left(\left(1-q_{1}\right)\left(1-q_{2}\right)+q_{2}\left(1-q_{3}\right)\right)\left(\left(1-q_{4}\right)\left(1-q_{2}\right)+q_{2}\left(1-q_{5}\right)\right)}{1+\left(1-q_{2}\right)\left(1-q_{1}\right)\left(2-q_{4}\right)+q_{2}\left(2-q_{5}\right)\left(1-q_{3}\right)}\end{cases}
$$

Let $H_{i}(i \epsilon\{1,2,3,4\})$ be the holding time of semi-Markov process at state $S_{i}$. Now we proceed to derive $E\left[H_{i}\right]$ :

$$
E\left[H_{1}\right]=E\left[t_{B}^{*}\right]+E\left[t_{I 1}^{*}\right]
$$

From Wald's theorem [6]

$$
E\left[t_{B}^{*}\right]=E\left[N_{p}\right] E\left[\frac{1}{\lambda_{i p}}\right]=\frac{\mu_{p}}{\lambda_{x}}
$$

$t_{I 1}^{*}=\min \left(t_{i p c}, t_{I}\right)$. If a packet arrives before the inactivity expire $t_{i p c}<t_{I}$, this means $t_{I 1}^{*}=t_{i p c}$, otherwise $t_{I 1}^{*}=t_{I}$ (next packet arrives after the inactivity has expired, $t_{i p c} \geq t_{I}$ ). Therefore,

$$
E\left[t_{I 1}^{*}\right]=P_{p c} E\left[\min \left(t_{i p c}, t_{I}\right)\right]
$$

We have

$$
\begin{aligned}
E\left[\min \left(t_{i p c}, t_{I}\right)\right] & =\int_{x=0}^{\infty} \operatorname{Pr}\left[\min \left(t_{i p c}, t_{I}\right)>x\right] d x \\
& =\int_{x=0}^{t_{I}} e^{-\lambda_{i p c} x} d x=\frac{1}{\lambda_{i p c}}\left[e^{-\lambda_{i p c} t_{I}}\right]
\end{aligned}
$$

Substitute (15) and (17) into (14)

$$
E\left[H_{1}\right]=\frac{\mu_{p}}{\lambda_{i p}}+\frac{P_{p c}}{\lambda_{i p c}}\left[1-e^{-\lambda_{i p c} t_{I}}\right]
$$

$E\left[H_{2}\right] . S_{2}$ contains a busy period $t_{B}^{*}$ and an intersession inactivity period $t_{I 2}^{*}$. Therefore,

$$
E\left[H_{2}\right]=E\left[t_{B}^{*}\right]+E\left[t_{I 2}^{*}\right]
$$

Similar to the derivation of $E\left[t_{I 1}^{*}\right], E\left[t_{I 2}^{*}\right]$ is

$$
\begin{aligned}
E\left[\min \left(t_{i s}, t_{I}\right)\right] & =\int_{x=0}^{\infty} \operatorname{Pr}\left[\min \left(t_{i s}, t_{I}\right)>x\right] d x \\
& =\int_{x=0}^{t_{I}} \operatorname{Pr}\left[t_{i s}>x\right] d x \\
& =\int_{x=0}^{t_{I}} e^{-\lambda_{i s} x} d x=\frac{1}{\lambda_{i s}}\left[e^{-\lambda_{i s} t_{I}}\right]
\end{aligned}
$$

Substitute (15) and (20) into (19)

$$
E\left[H_{2}\right]=\frac{\mu_{p}}{\lambda_{i p}}+\frac{P_{s}}{\lambda_{i s}}\left[1-e^{-\lambda_{i s} t_{I}}\right]
$$




\section{Sleep States $\mathrm{H}_{3}$ AND $\mathrm{H}_{4}$}

State $S_{3}$ comprises a Light Sleep period consisting of $N_{D S}$ DRX Short Cycles. We denote $N_{D S}$ as the total length of $t_{N}$ expressed in terms of the number of DRX Short Cycles. In this case the DRX Short Cycle Timer has expired and the UE enters into state $S_{4}$. The probability that a new packet call begins before $t_{N}$ expires results in $N_{D S}^{*}$, meaning $N_{D S}^{*}<N_{D S}$. Therefore, the mean holding time in state $S_{3}$ is:

$$
\begin{aligned}
E\left[H_{3}\right] & =E\left[N_{D S}\right] t_{D S} \\
& =\left(P_{34} N_{D S}+P_{31} E\left[N_{D S}^{i p c}\right]+P_{32} E\left[N_{D S}^{i s}\right]\right) t_{D S}
\end{aligned}
$$

Due to the memoryless property of the exponential $t_{i p c}$ and $t_{i s}, N_{D S}^{*}$ has a geometric distribution with mean $1 / P_{D S}$, where $P_{D S}$ is the probability that packets arrive during a DRX cycle and is derived as follows:

$$
\begin{aligned}
& E\left[N_{D S}^{i p c}\right]=\frac{P_{p c}}{\operatorname{Pr}\left[t_{i p c} \leq t_{D S}\right]}=\frac{P_{p c}}{1-e^{-\lambda_{i p c} t_{D S}}} \\
& E\left[N_{D S}^{i s}\right]=\frac{P_{s}}{\operatorname{Pr}\left[t_{i s} \leq t_{D S}\right]}=\frac{P_{s}}{1-e^{-\lambda_{i s} t_{D S}}}
\end{aligned}
$$

Then we substitute equations (9), (7), (8), (23) and (24) into (22):

$$
\begin{aligned}
E\left[H_{3}\right]= & {\left[\left(1-q_{2}\right)\left(1-q_{4}\right)+q_{2}\left(1-q_{5}\right)\right] N t_{D S} } \\
& +\left(\frac{q_{4}\left(1-q_{2}\right) P_{p c}}{1-e^{-\lambda_{i p c} t_{D S}}}+\frac{q_{2} q_{5} P_{s}}{1-e^{-\lambda_{i s} t_{D S}}}\right) t_{D S}
\end{aligned}
$$

State $S_{4}$ contains of Deep Sleep period consisting of State $n_{D L}$ Long DRX Cycles. Therefore $E\left[H_{4}\right]=E\left[n_{D L}\right] t_{D L}$ :

$$
E\left[H_{4}\right]=\left(\frac{P_{p c}}{1-e^{-\lambda_{i p c} t_{D L}}}+\frac{P_{s}}{1-e^{-\lambda_{i s} t_{D L}}}\right) t_{D L}
$$

\section{POWER SAVING FACTOR (PS)}

The power saving factor (PS) is equal to the probability that the semi-Markov process is at $S_{3}$ and $S_{4}$ in the steady state. Note that each DRX Short Cycle and each DRX Long Cycle contains a fixed On Duration $\tau$ so that it can listen to the paging information from the network. Therefore, the effective sleep duration is $t_{D S}^{\prime}=t_{D S}-\tau$ or $t_{D L}^{\prime}=t_{D L}-\tau$. Hence, the effective sleep time in both states $S_{3}$ and $S_{4}$ are derived as the following:

$$
\begin{gathered}
E\left[H_{3}^{\prime}\right]=\left(P_{34} N+P_{3,1} E\left[N_{D S}^{i p c}\right]+P_{3,2} E\left[N_{D S}^{i s}\right]\right) t_{D S}^{\prime} \\
=\left[\left(1-q_{2}\right)\left(1-q_{4}\right)+q_{2}\left(1-q_{5}\right)\right] N t_{D S}^{\prime} \\
+\left(\frac{q_{4}\left(1-q_{2}\right) P_{p c}}{1-e^{-\lambda_{i p c} t_{D S}}}+\frac{q_{2} q_{5} P_{s}}{1-e^{-\lambda_{i s} t_{D S}}}\right) t_{D S}^{\prime} \\
E\left[H_{4}^{\prime}\right]=\left(\frac{P_{p c}}{1-e^{-\lambda_{i p c} t_{D L}}}+\frac{P_{s}}{1-e^{-\lambda_{i s} t_{D L}}}\right) t_{D L}^{\prime}
\end{gathered}
$$

From Theorem 4.8.3 [9], we obtain $P S=\lim _{t \rightarrow \infty} \operatorname{Pr}$ [UE receiver is turned off at time $t$ ] for $P S$ to be obtain by:

$$
P S=\frac{\pi_{3} E\left[H_{3}^{\prime}\right]+\pi_{4} E\left[H_{4}^{\prime}\right]}{\sum_{i=1}^{4} \pi_{i} E\left[H_{i}\right]}
$$

Substituting Equations (13), (18), (22), (25), (26), (27) and (28) into Equation (29), we derive the closed-form equation for the power saving factor PS.

Next, we analyze the wake-up delay from the DRX. Whether we are in Deep Sleep or Light Sleep, a packet call transmission may begin in one of the sleep states. The probability that a packet call delivery starts during the $i^{\text {th }}$ DRX Cycle is in a fixed DRX Cycles:

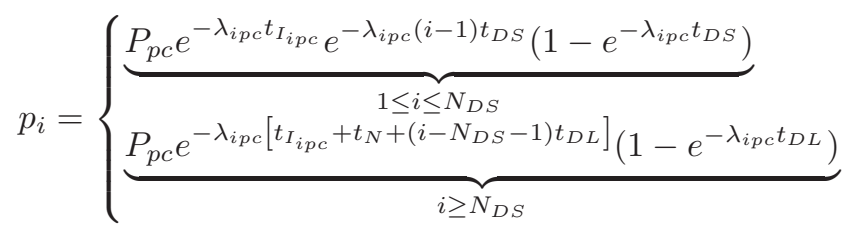




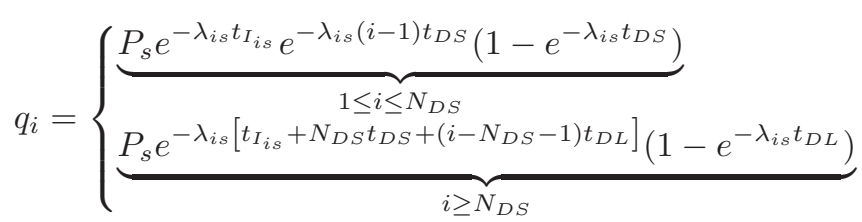

The packet call arrivals follow a Poisson distribution since the inter-packet call idle time and inter-session idle timer are random exponential distributed variables. Also, the arrival event are random observers to the sleep durations [13], [14], [17]. Therefore we have:

$$
\begin{aligned}
E[D]= & \sum_{i=1}^{N_{D S}} p_{i} \frac{t_{D S}}{2}+\sum_{i=N+1}^{\infty} p_{i} \frac{t_{D L}}{2} \\
& +\sum_{i=1}^{N_{D S}} q_{i} \frac{t_{D S}}{2}+\sum_{i=N+1}^{\infty} q_{i} \frac{t_{D L}}{2}
\end{aligned}
$$

Substituting Equation (30) into Equation (32), we derive the closed-form equation for the mean of wake-up delay $E[D]$.

\section{NumericAl Results}

The values of the parameters of the bursty packet data traffic model for the analytical model are as follows: $\lambda_{i p}=10, \lambda_{i p c}=1 / 30$, $\lambda_{i s}=1 / 2000, \mu_{p c}=5$, and $\mu_{p}=25$. We first analyse the effects of DRX parameters on DRX performance on the DRX Inactivity Timer $T_{I}$ in Figure 3. As $T_{I}$ increases, it is more likely that the next packet call starts before its expiration, which means lower transition probability for entering light or deep sleep state, respectively. Therefore, we observe a decrease in PS and D if $T_{I}$ increases. When $T_{N}$ increases, both PS and D decrease as well (Figure 4). It is more likely that the subsequent packet call deliveries happen before DRX Short Cycle Timer expires, and UE has less chance to enter the deep sleep period, so power saving performance becomes worse and wake-up delay performance gets better. Here we see the trade-off relationship between power saving factor and wake-up delay.

Next we will look at Figures 5 - 6, by focusing on the effects of the DRX Short Cycle $T_{D S}$ and the DRX Long Cycle $T_{D L}$. The power saving and delay shown in both Figures are increasing for both $T_{D S}$ and $T_{D L}$, which is due to the Sleep Cycles are longer and the "On Duration is fixed". The longer DRX Cycles translate into more effective sleep time per cycle, resulting in better power saving and a decrease in performance of the wake-up delay power saving.

From the Figures 3 - 6 there is a trade-off relationship between power saving factor and wake-up delay performance. When power saving performance is improved, wake-up delay performance will become worse. Therefore, DRX parameters should be selected carefully according to the tradeoff power saving factor and wake-up delay performance.

\section{CONCLUSION}

In this paper, we have taken an overview of LTE DRX mechanism with fixed/static DRX cycles and model it with bursty packet data traffic using a semi-Markov process. The analytical results show that LTE DRX will perform differently when adjusting the four DRX parameters. To verify the performance, four DRX parameters on output performance through the

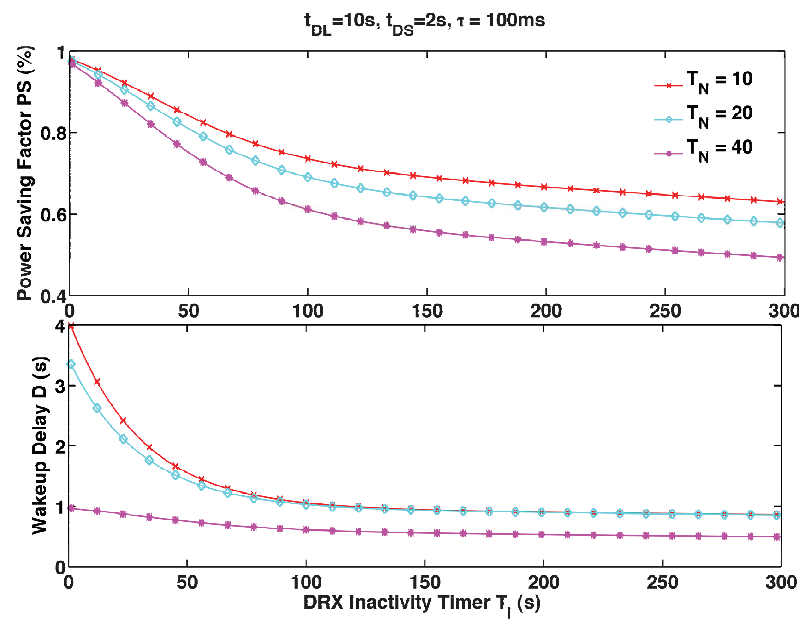

Fig. 3: (Top) LTE DRX Inactivity Timer on $T_{I}$ for Power. (Bottom) LTE DRX Inactivity Timer on $T_{I}$ for Delay. 


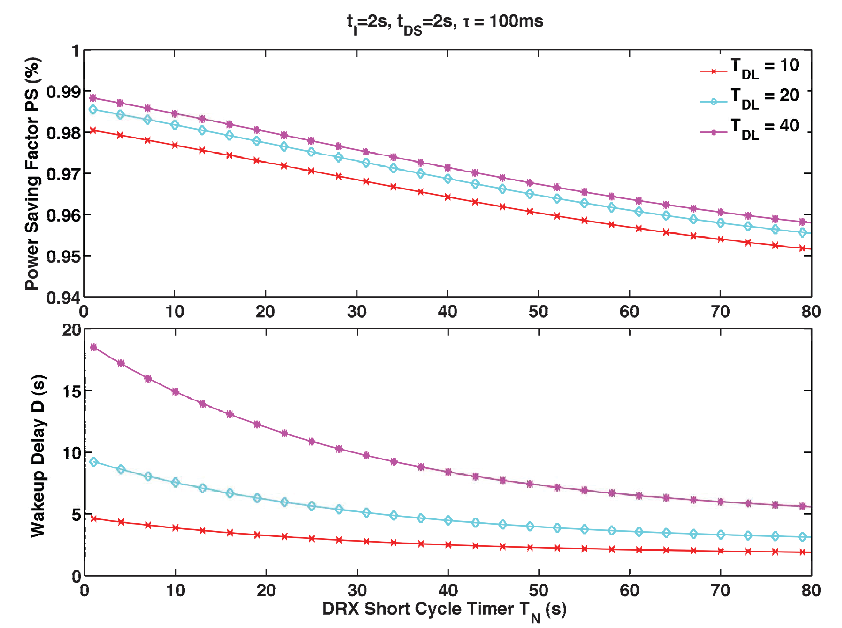

Fig. 4: (Top) LTE DRX Short Cycles on $T_{N}$ for Power. (Bottom) LTE DRX Short Cycles on $T_{N}$ for Delay.

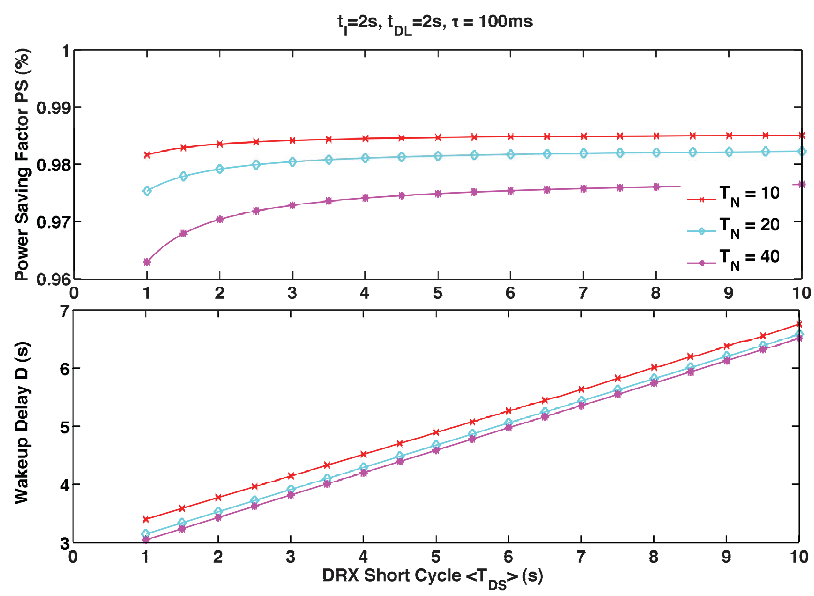

Fig. 5: (Top) LTE DRX Short Cycles on $T_{D S}$ for Power. (Bottom) LTE DRX Short Cycles on $T_{D S}$ for Delay.

analytical model in additional to a trade-off relationship between the power saving and wake-up delay performance was investigated. This work will help to select the best parameters when LTE DRX is implemented to achieve an efficient battery usage at a acceptable level of wake-up delay.

\section{ACKNOWLEDGMENT}

Scott Fowler was partially supported by the EC-FP7 Marie Curie CIG grant, Proposal number: 294182. Ranjeet S. Bhamber wishes to thank the financial support of Ministerio de Ciencia e Innovación through grant TEC2011-27314.

\section{REFERENCES}

[1] 3GPP TS 36.321. Medium Access Control (MAC) protocol specification, v10.2.0, (Release 10), March 2011.

[2] C. Bontu and E. Illidge. Drx mechanism for power saving in lte. Communications Magazine, IEEE, 47(6):48-55, June 2009.

[3] ETSI. Universal mobile telecommunications system (umts); selection procedures for the choice of radio transmission technologies of the umts. Technical Report UMTS 30.03, version 3.2.0, April 1998.

[4] Junxian Huang, Feng Qian, Alexandre Gerber, Z. Morley Mao, Subhabrata Sen, and Oliver Spatscheck. A close examination of performance and power characteristics of $4 \mathrm{~g}$ lte networks. In Proceedings of the 10th international conference on Mobile systems, applications, and services, MobiSys '12, 2012 .

[5] Lei Zhou, et al. Performance analysis of power saving mechanism with adjustable drx cycles in 3gpp lte. IEEE 68th Vehicular Technology Conference (VTC 2008), pages 1-5, September 2008 .

[6] Randolph Nelson. Probability, stochastic processes, and queueing theory: the mathematics of computer performance modeling. Springer-Verlag New York, Inc., New York, NY, USA, 1995.

[7] V. Paxson and S. Floyd. Wide area traffic: the failure of poisson modeling. Networking, IEEE/ACM Transactions on, $3(3): 226$-244, jun 1995.

[8] R. Mullner, et al. Contrasting open-loop and closed-loop power control performance in utran lte uplink by ue trace analysis. IEEE International Conference on Communications, (ICC '09), pages 1-6, June 2009.

[9] S. M. Ross. Stochastic Processes, 2nd Edition. John Wiley \& Sons, 1996. 


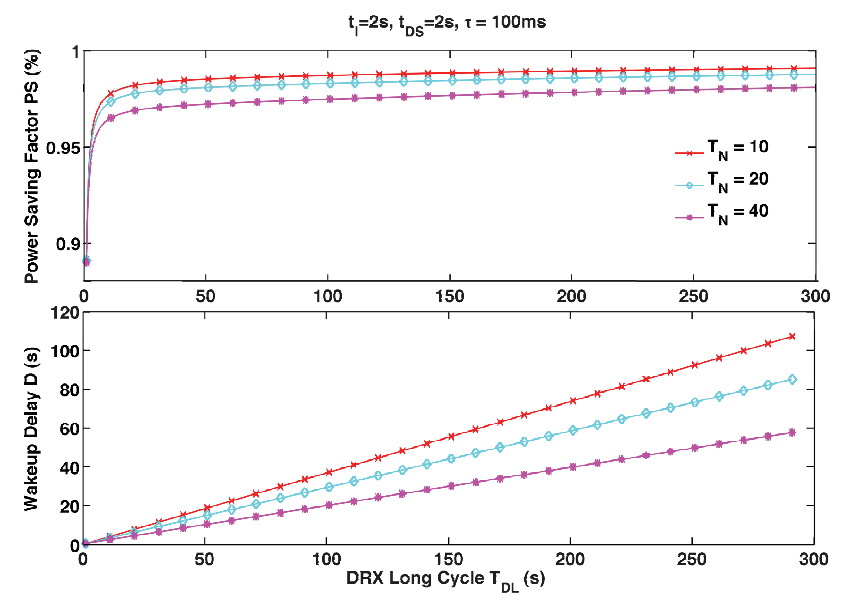

Fig. 6: (Top) LTE DRX Long Cycles on $T_{D L}$ for Power. (Bottom) LTE DRX Short Cycles on $T_{D L}$ for Delay.

[10] Scott Fowler and Ranjeet S. Bhamber and Abdelhamid Mellouk. Analysis of adjustable and fixed drx mechanism for power saving in lte/lte-advanced. IEEE International Conference on Communications (ICC 12), pages 10-15, June 2012.

[11] Shun-Ren Yang, et al. Modeling umts power saving with bursty packet data traffic. IEEE Transactions on Mobile Computing, 6(12):1398-1409, December 2007.

[12] Walter Willinger, et al. Self-similarity through high-variability: Statistical analysis of ethernet lan traffic at the source level. IEEE/ACM Transactions on Networking, 5(1):71-86, 1997.

[13] Y. Xiao. Energy saving mechanism in the ieee 802.16e wireless man. Communications Letters, IEEE, 9(7):595 - 597, july 2005.

[14] Yang Xiao. Performance analysis of an energy saving mechanism in the ieee 802.16e wireless man. Consumer Communications and Networking Conference, 2006. CCNC 2006. 3rd IEEE, 1:406 - 410, January 2006.

[15] Yuanye Wang, et al. Fixed frequency reuse for lte-advanced systems in local area scenarios. Vehicular Technology Conference, 2009. VTC Spring 2009. IEEE 69th, pages 1-5, April 2009.

[16] Yuanye Wang, et al. An interference aware dynamic spectrum sharing algorithm for local area lte-advanced networks. IEEE 70th Vehicular Technology Conference (VTC 2009), pages 1-5, September 2009.

[17] Yan Zhang, Yang Xiao, and V.C.M. Leung. Energy management analysis and enhancement in ieee 802.16e wirelessman. IEEE Transactions on Vehicular Technology, 58(7):3738-3752, September 2009. 